\title{
Solving the Poisson Equation by an Interval Difference Method of the Second Order
}

\author{
T. Hoffmann, A. Marciniak \\ Poznań University of Technology, Institute of Computing Science, Piotrowo 2, 60-965 Poznań, Poland \\ E-mail:tomaszhof@gmail.com, Andrzej.Marciniak@put.poznan.pl
}

Received: 15 January 2012; revised: 7 January 2013; accepted: 7 January 2013; published online: 22 January 2013

\begin{abstract}
The paper deals with an interval difference method for solving the Poisson equation based on the conventional central-difference method. We present the interval method in full details. The method is constructed in such a way that the exact solution is included in the interval solution obtained. Some numerical results obtained in floating-point interval arithmetic are also presented.
\end{abstract}

Key words: Poisson's equation, central-difference method, interval difference method

\section{INTRODUCTION}

In a number of our previous papers we have presented interval methods for solving the initial value problem in ordinary differential equations based on classical explicit and implicit Runge-Kutta methods and linear multistep methods (see e.g. [4-6]). All these works have been collected in [7]. It turns out that similar techniques for constructing interval methods can be applied to various problems in partial differential equations.

In this paper we present an interval difference method of the second order for solving the Poisson equation with the Dirichlet boundary conditions. The interval method is based on the conventional central-difference method. It has been found that at the mesh points the exact solution is within the obtained interval solution (see Sec. IV). Numerical experiments in floating-point interval arithmetic, presented in Sec. V, confirm the theoretical justifications.

\section{THE POISSON EQUATION}

An elliptic partial-differential equation, known as the Poisson equation, is of the form (see e.g. [1] or [3])

$$
\frac{\partial^{2} u}{\partial x^{2}}(x, y)+\frac{\partial^{2} u}{\partial y^{2}}(x, y)=f(x, y)
$$

We assume that function $f$ describes the input to the problem on plane region $R$ whose boundary will be denoted by $\Gamma$. Moreover, we assume that this function is continuous together with its partial derivatives up to the second order. Equations of this type arise naturally in the study of various time-independent problems such as:

- two-dimensional steady-state problems involving incompressible fluids,

- the potential energy of a point in a plane acted on by gravitational forces in the plane,

- the steady-state distribution of heat in a plane region.

To obtain a unique solution to the Poisson equation, additional constraints must be placed on the solution. Usually, we apply the Dirichlet boundary conditions, given by

$$
u(x, y)=\varphi(x, y)
$$

for all $(x, y)$ on $\Gamma$. In general, plane region $R$ may be arbitrary. Further, we will assume that $R$ is a rectangular:

$$
R=\{(x, y): 0<x<\alpha, 0<y<\beta\}
$$

Thus, our problem is to find $u=u(x, y)$ satisfying the partial- 
differential equation

$$
\begin{aligned}
& \frac{\partial^{2} u}{\partial x^{2}}(x, y)+\frac{\partial^{2} u}{\partial y^{2}}(x, y)=f(x, y), \\
& 0<x<\alpha, 0<y<\beta
\end{aligned}
$$

with the boundary conditions

$$
\left.u\right|_{\Gamma}=\varphi(x, y)=\left\{\begin{array}{lll}
\varphi_{1}(y) & \text { for } \quad x=0, \\
\varphi_{2}(x) & \text { for } \quad y=0, \\
\varphi_{3}(y) & \text { for } \quad x=\alpha \\
\varphi_{4}(x) & \text { for } \quad y=\beta,
\end{array}\right.
$$

where

$$
\begin{gathered}
\varphi_{1}(0)=\varphi_{2}(0), \varphi_{2}(\alpha)=\varphi_{3}(0), \\
\varphi_{3}(\beta)=\varphi_{4}(\alpha), \varphi_{4}(0)=\varphi_{1}(\beta), \\
\Gamma=\{(x, y): x=0, \alpha \text { and } 0 \leq y \leq \beta \\
\text { or } 0 \leq x \leq \alpha \text { and } y=0, \beta\} .
\end{gathered}
$$

\section{THE CENTRAL-DIFFERENCE METHOD}

The first step in any difference method is to choose integers $n$ and $m$, and define step sizes $h$ and $k$ by $h=\alpha / n$ and $k=\beta / m$. Partitioning the interval $[0, \alpha]$ into $n$ equal parts of width $h$ and the interval $[0, \beta]$ into $m$ equal parts of width $k$ provides a means of placing a grid on rectangle $R$ with mesh points $\left(x_{i}, y_{j}\right)=(i h, j k)$, where $i=0,1, \ldots, n$ and $j=0$, $1, \ldots, m$. Assuming that the fourth order partial derivatives of $u$ exist, for each mesh point in the interior of the grid we use the Taylor series in variable $x$ about $x_{i}$ to generate the central-difference formula

$$
\begin{aligned}
\frac{\partial^{2} u}{\partial x^{2}}\left(x_{i}, y_{j}\right) & =\frac{u\left(x_{i+1}, y_{j}\right)-2 u\left(x_{i}, y_{j}\right)+u\left(x_{i-1}, y_{j}\right)}{h^{2}} \\
& -\frac{h^{2}}{12} \frac{\partial^{4} u}{\partial x^{4}}\left(\xi_{i}, y_{j}\right),
\end{aligned}
$$

where $\xi_{i} \in\left(x_{i-1}, x_{i+1}\right)$, and the Taylor series in variable $y$ about $y_{j}$ to generate the central-difference formula

$$
\begin{aligned}
\frac{\partial^{2} u}{\partial y^{2}}\left(x_{i}, y_{j}\right) & =\frac{u\left(x_{i}, y_{j+1}\right)-2 u\left(x_{i}, y_{j}\right)+u\left(x_{i}, y_{j-1}\right)}{k^{2}} \\
& -\frac{k^{2}}{12} \frac{\partial^{4} u}{\partial y^{4}}\left(x_{i}, \eta_{j}\right),
\end{aligned}
$$

where $\eta_{j} \in\left(y_{j-1}, y_{j+1}\right)$. Using these formulas allows us to express the Poisson equation at the points $\left(x_{i}, y_{j}\right)$ as

$$
\begin{aligned}
& \frac{u\left(x_{i+1}, y_{j}\right)-2 u\left(x_{i}, y_{j}\right)+u\left(x_{i-1}, y_{j}\right)}{h^{2}} \\
+ & \frac{u\left(x_{i}, y_{j+1}\right)-2 u\left(x_{i}, y_{j}\right)+u\left(x_{i}, y_{j-1}\right)}{k^{2}}= \\
= & f\left(x_{i}, y_{j}\right)+\frac{h^{2}}{12} \frac{\partial^{4} u}{\partial x^{4}}\left(\xi_{i}, y_{j}\right)+\frac{k^{2}}{12} \frac{\partial^{4} u}{\partial y^{4}}\left(x_{i}, \eta_{j}\right), \\
& i=1,2, \ldots, n-1, j=1,2, \ldots, m-1,
\end{aligned}
$$

and the boundary conditions as

$$
\begin{array}{ll}
u\left(0, y_{j}\right)=\varphi_{1}\left(y_{j}\right) & \text { for each } j=0,1, \ldots, m, \\
u\left(x_{i}, 0\right)=\varphi_{2}\left(x_{i}\right) & \text { for each } i=1,2, \ldots, n-1, \\
u\left(\alpha, y_{j}\right)=\varphi_{3}\left(y_{j}\right) & \text { for each } j=0,1, \ldots, m, \\
u\left(x_{i}, \beta\right)=\varphi_{4}\left(x_{i}\right) & \text { for each } i=1,2, \ldots, n-1 .
\end{array}
$$

In the difference-equation form, this results in a method called the central-difference method, with local truncation error of order $O\left(h^{2}+k^{2}\right)$ that can be written in the following form (see e.g. [1] or [3]) :

$$
\begin{gathered}
k^{2} u_{i-1, j}+h^{2} u_{i, j-1}-2\left(h^{2}+k^{2}\right) u_{i, j} \\
+k^{2} u_{i+1, j}+h^{2} u_{i, j+1}=h^{2} k^{2} f\left(x_{i}, y_{j}\right), \\
i=1,2, \ldots, n-1, j=1,2, \ldots, m-1,
\end{gathered}
$$

and

$$
\begin{aligned}
u_{0, j}=\varphi_{1}\left(y_{j}\right) & \text { for each } j=0,1, \ldots, m, \\
u_{i, 0}=\varphi_{2}\left(x_{i}\right) & \text { for each } i=1,2, \ldots, n-1, \\
u_{n, j}=\varphi_{3}\left(y_{j}\right) & \text { for each } j=0,1, \ldots, m, \\
u_{i, m}=\varphi_{4}\left(x_{i}\right) & \text { for each } i=1,2, \ldots, n-1,
\end{aligned}
$$

where $u_{i, j}$ approximates $\mathrm{u}\left(x_{i}, y_{j}\right)$.

The equations (5), together with (6), are an $(n-1)(m-1)$ system of linear equations in $(n-1)(m-1)$ unknowns $u_{i, j}$ being the approximations to $u\left(x_{i}, y_{j}\right)$ for the interior mesh points.

\section{AN INTERVAL DIFFERENCE METHOD OF THE SECOND ORDER}

Let us consider the following central-difference formulas:

$$
\begin{aligned}
\frac{\partial^{2} u}{\partial x^{2}} & =\frac{u(x-h, y)-2 u(x, y)+u(x+h, y)}{h^{2}} \\
& -\frac{h^{2}}{12} \frac{\partial^{4} u}{\partial x^{4}}(\xi, y), \xi \in(x-h, x+h), \\
\frac{\partial^{2} u}{\partial y^{2}} & =\frac{u(x, y-k)-2 u(x, y)+u(x, y+k)}{k^{2}} \\
& -\frac{h^{2}}{12} \frac{\partial^{4} u}{\partial y^{4}}(x, \eta), \eta \in(y-k, y+k),
\end{aligned}
$$

and try to find intervals containing $\frac{\partial^{4} u}{\partial x^{4}}(\xi, y)$ and $\frac{\partial^{4} u}{\partial y^{4}}(x, \eta)$.

From the Poisson equation it follows that 


$$
\begin{aligned}
& \frac{\partial^{4} u}{\partial x^{4}}(x, y)=\frac{\partial^{2} f}{\partial x^{2}}(x, y)-\frac{\partial^{4} u}{\partial x^{2} \partial y^{2}}(x, y), \\
& \frac{\partial^{4} u}{\partial y^{4}}(x, y)=\frac{\partial^{2} f}{\partial y^{2}}(x, y)-\frac{\partial^{4} u}{\partial y^{2} \partial x^{2}}(x, y) .
\end{aligned}
$$

Let

$$
\frac{\partial^{4} u}{\partial x^{2} \partial y^{2}}(x, y)=\frac{\partial^{4} u}{\partial y^{2} \partial x^{2}}(x, y),
$$

and let us assume that there exists a constant $M$ such that

$$
\left|\frac{\partial^{4} u}{\partial x^{2} \partial y^{2}}\right| \leq M \text { for all } 0 \leq x \leq \alpha \text { and } 0 \leq y \leq \beta .
$$

In practice, it can be difficult to determine the constant $M$. Sometimes it is possible to establish a value of $M$ on the basis of the considered physical problem, but it is rather a seldom case. Thus, we propose to find this value by an approximation. On the basis of (7) we have

$$
\begin{aligned}
& \frac{\partial^{2}}{\partial y^{2}}\left(\frac{\partial^{2} u}{\partial x^{2}}\right)= \\
& =\frac{u(x-h, y-k)-2 u(x-h, y)+u(x-h, y+k)}{h^{2} k^{2}} \\
& -2 \frac{u(x, y-k)-2 u(x, y)+u(x, y+k)}{h^{2} k^{2}} \\
& +\frac{u(x+h, y-k)-2 u(x+h, y)+u(x+h, y+k)}{h^{2} k^{2}} \\
& -\frac{k^{2}}{12 h^{2}}\left[\frac{\partial^{4} u}{\partial y^{4}}\left(x-h, \eta_{1}\right)+\frac{\partial^{4} u}{\partial y^{4}}\left(x, \eta_{2}\right)+\frac{\partial^{4} u}{\partial y^{4}}\left(x+h, \eta_{3}\right)\right] \\
& -\frac{h^{2}}{12} \frac{\partial^{2}}{\partial y^{2}}\left[\frac{\partial^{4} u}{\partial x^{4}}(\xi, y)\right]
\end{aligned}
$$

and

$$
\begin{aligned}
& \frac{\partial^{2}}{\partial x^{2}}\left(\frac{\partial^{2} u}{\partial y^{2}}\right)= \\
& =\frac{u(x-h, y-k)-2 u(x, y-k)+u(x+h, y-k)}{h^{2} k^{2}} \\
& -2 \frac{u(x-h, y)-2 u(x, y)+u(x+h, y)}{h^{2} k^{2}} \\
& +\frac{u(x-h, y+k)-2 u(x, y+k)+u(x+h, y+k)}{h^{2} k^{2}} \\
& -\frac{h^{2}}{12 k^{2}}\left[\frac{\partial^{4} u}{\partial x^{4}}\left(\xi_{1}, y-k\right)+\frac{\partial^{4} u}{\partial x^{4}}\left(\xi_{2}, y\right)+\frac{\partial^{4} u}{\partial x^{4}}\left(\xi_{3}, y+k\right)\right] \\
& -\frac{k^{2}}{12} \frac{\partial^{2}}{\partial x^{2}}\left[\frac{\partial^{4} u}{\partial y^{4}}(x, \eta)\right],
\end{aligned}
$$

where $\xi, \xi_{1}, \xi_{2}, \xi_{3} \in(x-h, x+h), \eta, \eta_{1}, \eta_{2}, \eta_{3} \in(y-$ $k, y+k)$. If $h$ and $k$ are sufficiently small and the fourth order partial derivatives are not very large, then from the above equations it follows that

$$
\begin{aligned}
& \frac{\partial^{2}}{\partial y^{2}}\left(\frac{\partial^{2} u}{\partial x^{2}}\right) \approx \\
& \approx \frac{u(x-h, y-k)-2 u(x-h, y)+u(x-h, y+k)}{h^{2} k^{2}} \\
& -2 \frac{u(x, y-k)-2 u(x, y)+u(x, y+k)}{h^{2} k^{2}} \\
& +\frac{u(x+h, y-k)-2 u(x+h, y)+u(x+h, y+k)}{h^{2} k^{2}}
\end{aligned}
$$

and

$$
\begin{aligned}
& \frac{\partial^{2}}{\partial x^{2}}\left(\frac{\partial^{2} u}{\partial y^{2}}\right) \approx \\
& \approx \frac{u(x-h, y-k)-2 u(x, y-k)+u(x+h, y-k)}{h^{2} k^{2}} \\
& -2 \frac{u(x-h, y)-2 u(x, y)+u(x+h, y)}{h^{2} k^{2}} \\
& +\frac{u(x-h, y+k)-2 u(x, y+k)+u(x+h, y+k)}{h^{2} k^{2}} .
\end{aligned}
$$

The right-hand sides of the above approximations are equal and therefore we propose to approximate the constant $M$ (given by (8)) as follows:

$$
\begin{aligned}
& M \approx \frac{1.5}{h^{2} k^{2}} \max _{\substack{i=1,2, \ldots, n-1 \\
j=1,2, \ldots, m-1}} \mid 4 u_{i, j} \\
& -2\left(u_{i-1, j}+u_{i, j-1}+u_{i, j+1}+u_{i+1, j}\right), \\
& +u_{i-1, j-1}+u_{i-1, j+1}+u_{i+1, j-1}+u_{i+1, j+1} \mid,
\end{aligned}
$$

where $u_{i j}$ are obtained by the conventional central-difference method (5)-(6), and the coefficients 1.5 (instead of 1.0) stays that we take the value $50 \%$ greater.

Let $\Psi(X, Y)$ and $\Omega(X, Y)$ denote interval extensions of $\frac{\partial^{2} f}{\partial x^{2}}(x, y)$ and $\frac{\partial^{2} f}{\partial y^{2}}(x, y)$, respectively (for the definition of interval extension see e.g. [2], [8] or [9]). According to (8), it is obvious that we have

$$
\frac{\partial^{4} u}{\partial x^{4}}(\xi, y) \in \Psi(X+[-h, h], Y)+[-M, M]
$$

and

$$
\frac{\partial^{4} u}{\partial y^{4}}(x, \eta) \in \Omega(X, Y+[-k, k])+[-M, M],
$$

and these relations are true for each mesh point $\left(x_{i}, y_{j}\right) \in\left(X_{i}\right.$, $Y_{j}$ ). If we recall the Poisson equation at the mesh points (3), it is easy now to write an interval equivalent to this equation. We have

$$
\begin{aligned}
& k^{2} U_{i-1, j}+h^{2} U_{i, j-1}-2\left(h^{2}+k^{2}\right) U_{i, j} \\
& +k^{2} U_{i+1, j}+h^{2} U_{i, j+1}= \\
& =h^{2} k^{2}\left\{F_{i, j}+\frac{1}{12}\left[h^{2} \Psi\left(X_{i}+[-h, h], Y_{j}\right)\right.\right. \\
& \left.\left.+k^{2} \Omega\left(X_{i}, Y_{j}+[-k, k]\right)+\left(h^{2}+k^{2}\right)[-M, M]\right]\right\}, \\
& i=1,2, \ldots, n-1, j=1,2, \ldots, m-1,
\end{aligned}
$$


where $F_{i, j}=F\left(X_{i}, Y_{j}\right)$, and where

$$
\begin{aligned}
U_{0, j} & =\Phi_{1}\left(Y_{j}\right) & & \text { for each } j=0,1, \ldots, m, \\
U_{i, 0} & =\Phi_{2}\left(X_{i}\right) & & \text { for each } i=1,2, \ldots, n-1, \\
U_{n, j} & =\Phi_{3}\left(Y_{j}\right) & & \text { for each } j=0,1, \ldots, m, \\
U_{i, m} & =\Phi_{4}\left(X_{i}\right) & & \text { for each } i=1,2, \ldots, n-1 .
\end{aligned}
$$

$\left(\Phi_{1}(Y), \Phi_{2}(X), \Phi_{3}(Y)\right.$ and $\Phi_{4}(X)$ denote interval extensions of the functions $\phi_{1}(y), \phi_{2}(x), \phi_{3}(y)$ and $\phi_{4}(x)$, respectively.)

Since in the Poisson equation at the mesh points we have approximated all the values by their interval extensions and by other intervals containing these values, we can expect that $u\left(x_{i}, y_{j}\right) \in U_{i, j}(i=0,1, \ldots, n ; j=0,1, \ldots, m)$, i.e. at the mesh points the exact solution is within the obtained interval solution. In our interval method the term

$$
\begin{aligned}
& \frac{1}{12}\left[h^{2} \Psi\left(X_{i}+[-h, h], Y_{j}\right)\right. \\
+ & \left.k^{2} \Omega\left(X_{i}, Y_{j}+[-k, k]\right)+\left(h^{2}+k^{2}\right)[-M, M]\right]
\end{aligned}
$$

is very important as it guarantees that the error of the method will be included in the interval solution obtained.

To find the interval solution at the interior mesh points of the grid we have to solve the $(n-1)(m-1)$ system of interval linear equations (10) in $(n-1)(m-1)$ unknowns $U_{i, j}$. This system can be written in the form

$$
\mathbf{A U}=\mathbf{Q}
$$

where

$$
\begin{aligned}
\mathbf{A} & =\left[\begin{array}{ccccccc}
\mathbf{B} & \mathbf{C} & \mathbf{0} & \ldots & \mathbf{0} & \mathbf{0} & \mathbf{0} \\
\mathbf{C} & \mathbf{B} & \mathbf{C} & \ldots & \mathbf{0} & \mathbf{0} & \mathbf{0} \\
\mathbf{0} & \mathbf{C} & \mathbf{B} & \ldots & \mathbf{0} & \mathbf{0} & \mathbf{0} \\
\vdots & \vdots & \vdots & \ddots & \vdots & \vdots & \vdots \\
\mathbf{0} & \mathbf{0} & \mathbf{0} & \ldots & \mathbf{B} & \mathbf{C} & \mathbf{0} \\
\mathbf{0} & \mathbf{0} & \mathbf{0} & \ldots & \mathbf{C} & \mathbf{B} & \mathbf{C} \\
\mathbf{0} & \mathbf{0} & \mathbf{0} & \ldots & \mathbf{0} & \mathbf{C} & \mathbf{B}
\end{array}\right], \\
\mathbf{U} & =\left[\begin{array}{c}
\mathbf{U}_{1} \\
\mathbf{U}_{2} \\
\mathbf{U}_{3} \\
\vdots \\
\mathbf{U}_{n-3} \\
\mathbf{U}_{n-2} \\
\mathbf{U}_{n-1}
\end{array}\right], \quad \mathbf{Q}=\left[\begin{array}{c}
\mathbf{Q}_{1} \\
\mathbf{Q}_{2} \\
\mathbf{Q}_{3} \\
\vdots \\
\mathbf{Q}_{n-3} \\
\mathbf{Q}_{n-2} \\
\mathbf{Q}_{n-1}
\end{array}\right]
\end{aligned}
$$

$$
\begin{gathered}
\mathbf{U}_{i}=\left[\begin{array}{c}
U_{i, 1} \\
U_{i, 2} \\
\vdots \\
U_{i, m-1}
\end{array}\right], \mathbf{Q}_{i}=\left[\begin{array}{c}
Q_{i, 1} \\
Q_{i, 2} \\
\vdots \\
Q_{i, m-1}
\end{array}\right], \\
i=1,2, \ldots, n-1, \\
\mathbf{B}=\left[\begin{array}{ccccccc}
\gamma & h^{2} & 0 & \ldots & 0 & 0 & 0 \\
h^{2} & \gamma & h^{2} & \ldots & 0 & 0 & 0 \\
0 & h^{2} & \gamma & \ldots & 0 & 0 & 0 \\
\vdots & \vdots & \vdots & \ddots & \vdots & \vdots & \vdots \\
0 & 0 & 0 & \ldots & \gamma & h^{2} & 0 \\
0 & 0 & 0 & \ldots & h^{2} & \gamma & h^{2} \\
0 & 0 & 0 & \ldots & 0 & h^{2} & \gamma
\end{array}\right],
\end{gathered}
$$

$$
\mathbf{C}=\left[\begin{array}{ccccc}
k^{2} & 0 & \cdots & 0 & 0 \\
0 & k^{2} & \cdots & 0 & 0 \\
\vdots & \vdots & \ddots & \vdots & \vdots \\
0 & 0 & \cdots & k^{2} & 0 \\
0 & 0 & \cdots & 0 & k^{2}
\end{array}\right]
$$

$\operatorname{dim} \mathbf{B}=\operatorname{dim} \mathbf{C}=(m-1) \times(m-1)$,

$$
\begin{aligned}
Q_{1,1}= & \Lambda_{1,1}-k^{2} \Phi_{1}([k, k])-h^{2} \Phi_{2}([h, h]), \\
Q_{1, j}= & \Lambda_{1, j}-k^{2} \Phi_{1}([j k, j k]), \\
& j=2,3, \ldots, m-2 \\
Q_{1, m-1}= & \Lambda_{1, m-1}-k^{2} \Phi_{1}([(m-1) k,(m-1) k]) \\
- & h^{2} \Phi_{4}([h, h]) \\
Q_{i, 1}= & \Lambda_{i, 1}-h^{2} \Phi_{2}([i h, i h]), \\
& i=2,3, \ldots, n-2, \\
Q_{i, j}= & \Lambda_{i, j}, \\
& i=2,3, \ldots, n-2, j=2,3, \ldots, m-2, \\
Q_{i, m-1}= & \Lambda_{i, m-1}-h^{2} \Phi_{4}([i h, i h]), \\
& i=2,3, \ldots, n-2, \\
Q_{n-1,1}= & \Lambda_{n-1,1}-h^{2} \Phi_{2}([(n-1) h,(n-1) h]) \\
- & k^{2} \Phi_{3}([k, k]), \\
Q_{n-1, j}= & \Lambda_{n-1, j}-k^{2} \Phi_{3}([j k, j k]), \\
& j=2,3, \ldots, m-2, \\
= & \Lambda_{n-1, m-1}-k^{2} \Phi_{3}([(m-1) k,(m-1) k]) \\
- & h^{2} \Phi_{4}([(n-1) h,(n-1) h]),
\end{aligned}
$$

where

$$
\begin{aligned}
\Lambda_{i, j} & =h^{2} k^{2}\left\{F_{i, j}+\frac{1}{12}\left[h^{2} \Psi\left(X_{i}+[-h, h], Y_{j}\right)\right.\right. \\
& \left.+k^{2} \Omega\left(X_{i}, Y_{j}+[-k, k]+\left(h^{2}+k^{2}\right)[-M, M]\right]\right\} .
\end{aligned}
$$


In the conventional central-difference method to solve the adequate system of linear equations we usually use an iterative method (due to the fact that the system is very large and has the special form of the matrix), e.g. the Gauss-Seidel method. We do not recommend using any iterative method to solve the system of interval linear equations (12), occurring in the presented interval method, because of additional errors that the iterative method can cause.

Taking into account the special form of the system matrix (with a lot of elements equal to 0 ) we propose to use the Crout method which consists in factoring the matrix $\mathbf{A}$ in terms of a lower-triangular matrix $\mathbf{L}$ and an upper-triangular matrix $\mathbf{R}$ (usually denoted by $\mathbf{U}$, but in this paper we use the letter $\mathbf{U}$ for another purpose). Matrices $\mathbf{L}$ and $\mathbf{R}$ are band matrices of the following forms:
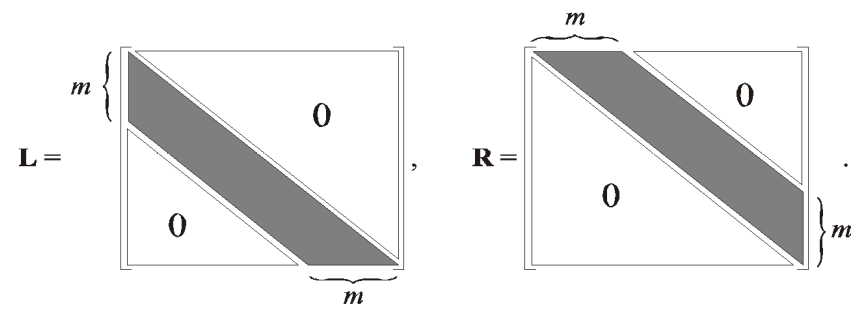

To find the elements of $\mathbf{L}$ and $\mathbf{R}$ we have the following algorithm:

$$
\begin{aligned}
& \text { for } i:=1 \text { to }(m-1)(n-1) \text { do } \\
& r_{i i}:=1 \\
& l_{i i}:=a_{i i}-\sum_{s=1}^{i-1} l_{i s} r_{s i} \\
& \text { for } j:=1 \text { to } \min \{m+i-1,(m-1)(n-1)\} \text { do } \\
& \quad r_{i j}:=\frac{a_{i j}-\sum_{s=1}^{i-1} l_{i s} r_{s j}}{l_{i i}} \\
& \quad l_{j i}:=a_{j i}-\sum_{s=1}^{i-1} l_{j s} r_{s i} \\
& \text { end for } \\
& \text { end for }
\end{aligned}
$$

\section{end for}

where

$$
\begin{aligned}
a_{i i}=\gamma, & i=1,2, \ldots,(m-1)(n-1), \\
a_{i, i+1}=h^{2}, & i=1,2, \ldots,(m-1)(n-1)-1 \text { and } \\
& i \neq s(m-1), \\
& \text { where } s=1,2, \ldots, n-2, \\
a_{i, i-1}=h^{2}, & i=2,3, \ldots,(m-1)(n-1) \text { and } \\
& i \neq s(m-1)+1, \\
& \text { where } s=1,2, \ldots, n-2, \\
a_{i, i-1+m}=k^{2}, & i=1,2, \ldots,(m-1)(n-2), \\
a_{i, i+1-m}=k^{2}, & i=m, m+1, \ldots,(m-1)(n-1), \\
a_{j i}=0, & \text { for others } j \text { and } i .
\end{aligned}
$$

The system $\mathbf{A} \mathbf{U}=\mathbf{Q}$ can be then transformed into the system $\mathbf{L R U}=\mathbf{Q}$. Since $\mathbf{L}$ is a lower-triangular matrix, the forward substitution solves the system $\mathbf{L Z}=\mathbf{Q}$, and since $\mathbf{R}$ is an upper-triangular matrix, the backward substitution solves the system $\mathbf{R U}=\mathbf{Z}$.

\section{NUMERICAL EXPERIMENTS}

In the examples presented in this section all calculations have been performed using our IntervalArithmetic unit (version 2.13, 2009) written in the Delphi Pascal language and presented in [7]. The IntervalArithmetic unit makes it possible to:

- represent any input data in the form of a machine interval (the ends of this interval are equal or are two subsequent machine numbers),

- perform all calculations in floating-point interval arithmetic,

- use some standard interval functions,

- give results in the form of proper intervals (if the ends of an interval are not the same machine numbers, the difference is shown in the output).

For solving the adequate systems of interval linear equations the Crout factorization has been used in each case.

Example 1. Let us consider the boundary value problem of the form

$$
\frac{\partial^{2} u}{\partial x^{2}}+\frac{\partial^{2} u}{\partial y^{2}}=0, u=u(x, y), 0<x<1,0<y<1,
$$

where

$$
\left.u\right|_{\Gamma}=\varphi(x, y)=\left\{\begin{array}{l}
\varphi_{1}(y)=\cos (3 y) \text { for } x=0 \\
\varphi_{2}(x)=\exp (3 x) \text { for } y=0 \\
\varphi_{3}(y)=\exp (3) \cos (3 y) \text { for } x=1 \\
\varphi_{4}(x)=\exp (3 x) \cos (3) \text { for } y=1
\end{array}\right.
$$

The exact solution of this problem is as follows:

$$
u(x, y)=\exp (3 x) \cos (3 y)
$$

The solution of the problem (13)-(15) is presented in Fig. 1, while in Fig. 2 the relative errors obtained in the conventional central-difference method on the grid $x=0.5$ and $y=0.5$ are given.

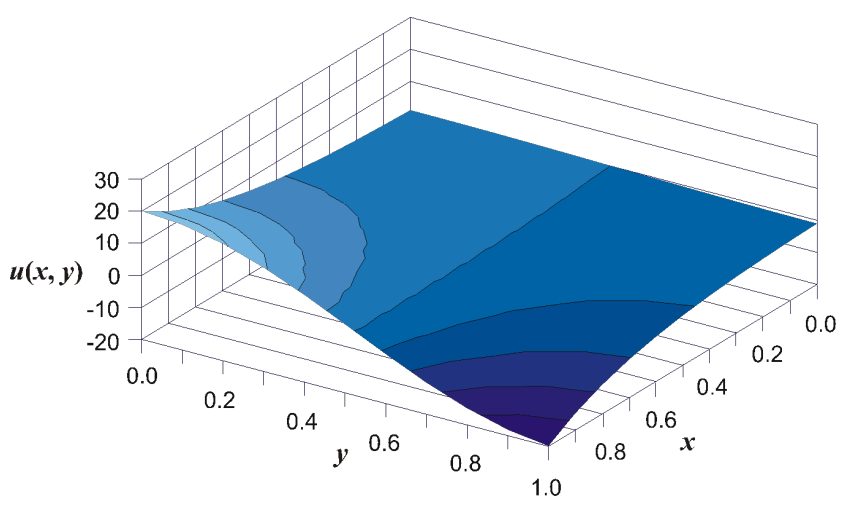

Fig. 1. The solution of the problem (13)-(15) 
Tab. 1. The interval solutions of the problem $(13)-(15)$ at $(0.5,0.5)\left(u_{\text {exact }}(0.5,0.5) \approx 0.31702214358044366\right)$

\begin{tabular}{ccccc}
\hline \hline$n=m$ & $u_{\text {conv }}(0.5,0.5)$ & $U(0.5,0.5)$ & Width of $U$ \\
\hline 20 & 0.31780279965243506 & {$[0.26795781801796551,0.36764778128690462]$} & 0.0997 \\
30 & 0.31736946637561425 & {$[0.29519196542239099,0.33954696732883751]$} & 0.0444 \\
40 & 0.31721758461727955 & {$[0.30473796887028390,0.32969720036427600]$} & 0.0250 \\
50 & 0.31714724718403603 & {$[0.30915887832531316,0.32513561604275890]$} & 0.0160 \\
60 & 0.31710902913708347 & {$[0.31156101681974897,0.32265704145441798]$} & 0.0111 \\
70 & 0.31708598143593676 & {$[0.31300965415668941,0.32116230871518411]$} & 0.0082 \\
80 & 0.31707102121236846 & {$[0.31394996555028855,0.32019207687444838]$} & 0.0062 \\
\hline 90 & 0.31706076390493784 & {$[0.31459468091091084,0.31952684689896485]$} & 0.0049 \\
\hline 100 & 0.31705342660012054 & {$[0.31505586246198510,0.31905099073825598]$} & 0.0040 \\
\hline \hline
\end{tabular}

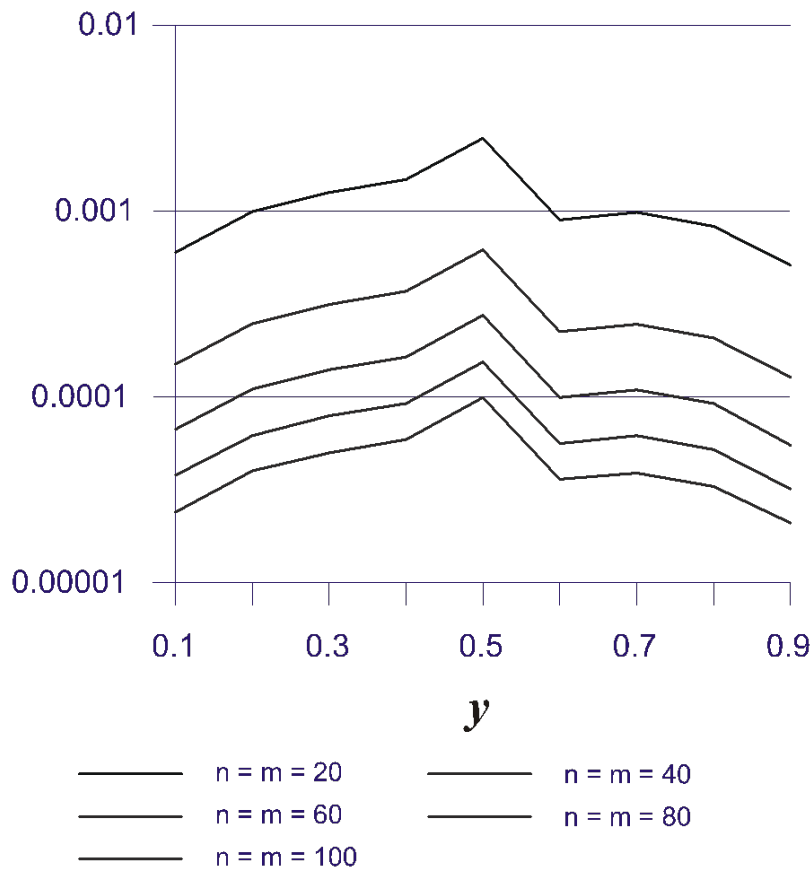

(a)

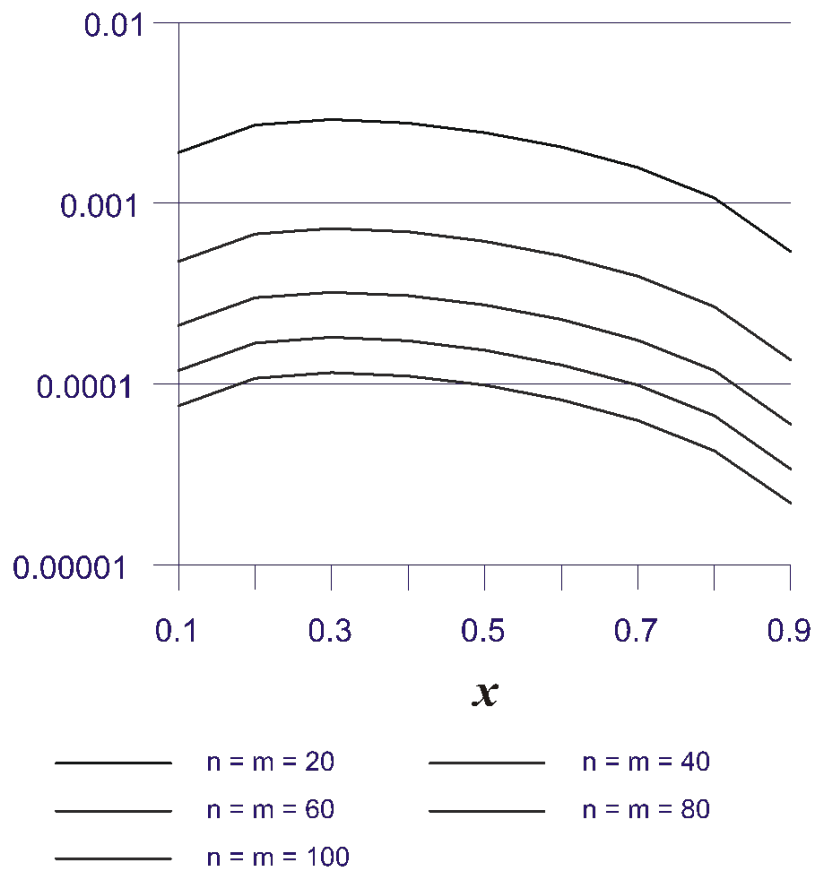

(b)

Fig. 2. The relative errors of $u(x, y)$ in the conventional central-difference method on the grids (a) $x=0.5$ and (b) $y=0.5$ 
To find the solution by our interval method, we should first determine the constant $M$ given by (8). For the problem (13)-(15) we have

$$
\left|\frac{\partial^{4} u}{\partial x^{2} \partial y^{2}}\right|_{\substack{x \in[0,1] \\ y \in[0,1]}} \leq 81 \exp (3)<1627=M .
$$

The interval solutions obtained at the mesh point $(0.5,0.5)$ for different values of $n=m$, together with their widths, are given in Table 1 . The widths of interval solutions on the grid $X=0.5$ are also presented in Fig. 3 (on the grid $Y=0.5$ the widths are the same). Let us note that at each mesh point we have $u(x, y) \in U(X, Y)$, i.e. the exact solution belongs to the obtained interval solutions.

In Fig. 4 we present the times of calculations as the functions of $n=m$ in the conventional and interval centraldifference methods. These times refer the Intel Core2 Duo T5450 1.67GHz processor and $2 \mathrm{~GB}$ computer RAM. Although the shapes of both curves are similar, one should notice that in the conventional central-difference method the time is measured in minutes, while in the interval one it is measured in hours and is approximately 20 times longer.

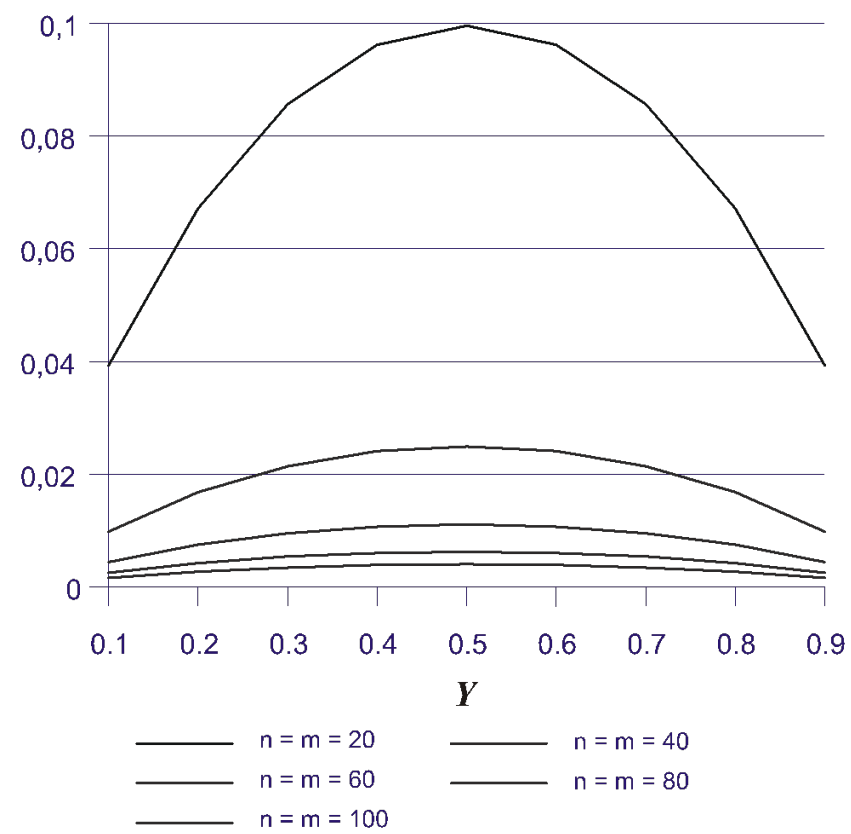

Fig. 3. The widths of interval solutions $U(X, Y)$ of the problem (13)-(15) on the grid $X=0.5$

Example 2. Let us take into account the following boundary value problem:

$$
\begin{aligned}
& \frac{\partial^{2} u}{\partial x^{2}}+\frac{\partial^{2} u}{\partial y^{2}}=-2 \pi^{2} \sin (\pi x) \sin (\pi y), \\
u= & u(x, y), 0<x<1,0<y<1,\left.u\right|_{\Gamma}=0 .
\end{aligned}
$$

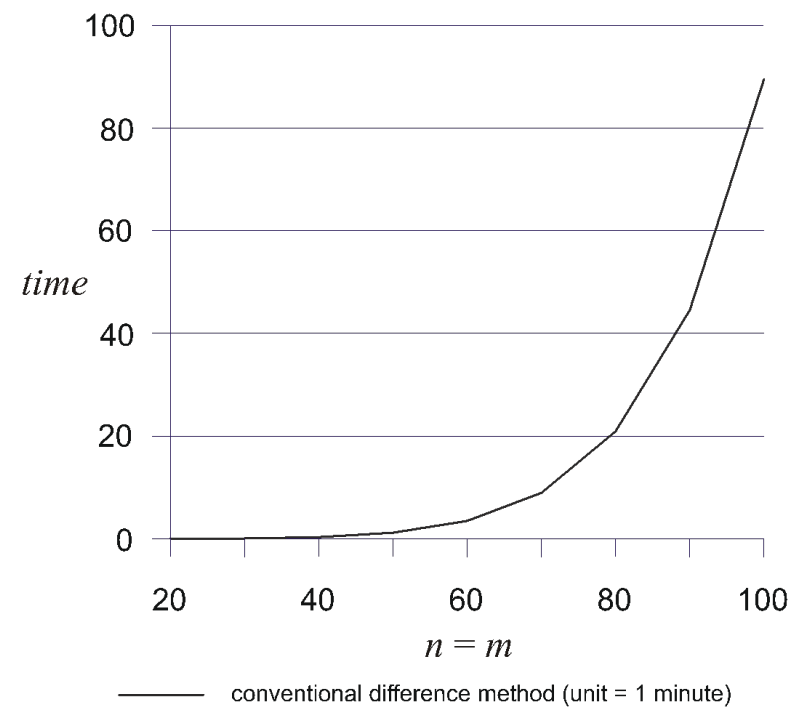

(a)

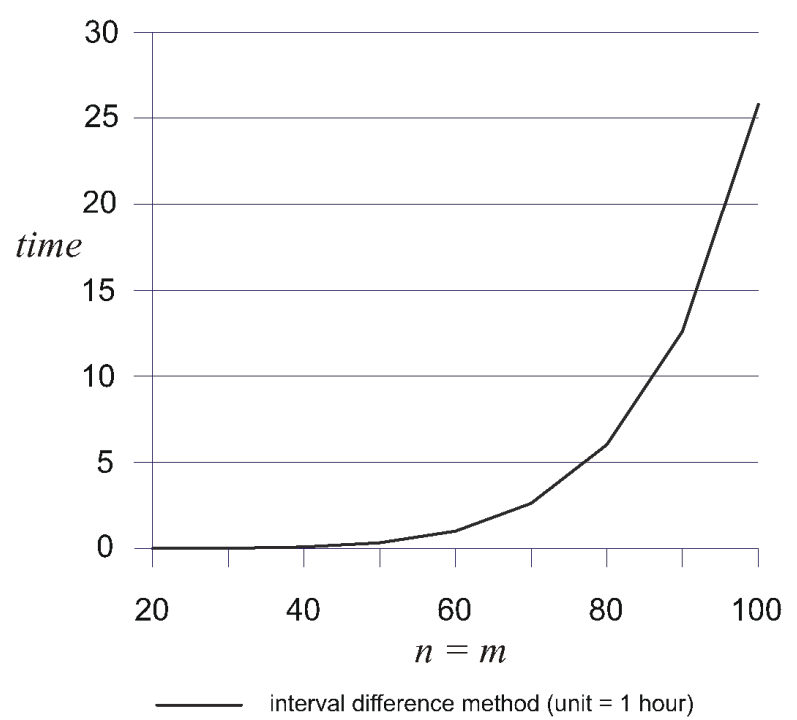

(b)

Fig. 4. The times of calculations in the conventional and interval central-difference methods

The exact solution of (15) is given by (see Fig. 5)

$$
u(x, y)=\sin (\pi x) \sin (\pi y) .
$$

For the constant $M$ in the method (9) we have

$$
\left|\frac{\partial^{4} u}{\partial x^{2} \partial y^{2}}\right|_{\substack{x \in[0,1] \\ y \in[0,1]}} \leq \pi^{4}<98.5=M .
$$

In Table 2 we present the interval solutions obtained at the mesh point $(0.5,0.5)$ for different values of $n=m$. The widths of interval solutions are also given in this table and in Fig. 6. As in Example 1, at each mesh point we have $u(x, y) \in U(X, Y)$. 
Tab. 2. The interval solutions of the problem $(15)$ at $(0.5,0.5)\left(u_{\text {exact }}(0.5,0.5) \approx 1\right)$

\begin{tabular}{ccccc}
\hline \hline$n=m$ & $u_{\text {conv }}(0.5,0.5)$ & $U(0.5,0.5)$ & Width of $U$ \\
\hline 20 & 1.0020587067645337 & {$[0.99427253616548267,1.0033273360116428]$} & 0.00905 \\
30 & 1.0009143535530669 & {$[0.99747509861203634,1.0009143535530671]$} & 0.00366 \\
40 & 1.0005142004781495 & {$[0.99858835887907646,1.0005142004781498]$} & 0.00195 \\
50 & 1.0003290517629385 & {$[0.99910517543983971,1.0003052815215404]$} & 0.00120 \\
60 & 1.0002284943854706 & {$[0.99938436578314373,1.0001944774813223]$} & 0.00081 \\
70 & 1.0001678673205915 & {$[0.99955133118044769,1.0001337049950954]$} & 0.00058 \\
80 & 1.0001285203835441 & {$[0.99965883352361963,1.0000971066859084]$} & 0.00044 \\
\hline 90 & 1.0001015453263438 & {$[0.99973199107949138,1.0000734967780457]$} & 0.00034 \\
100 & 1.0000822507622135 & {$[0.99978398196237121,1.0000574415620096]$} & 0.00027 \\
\hline \hline
\end{tabular}

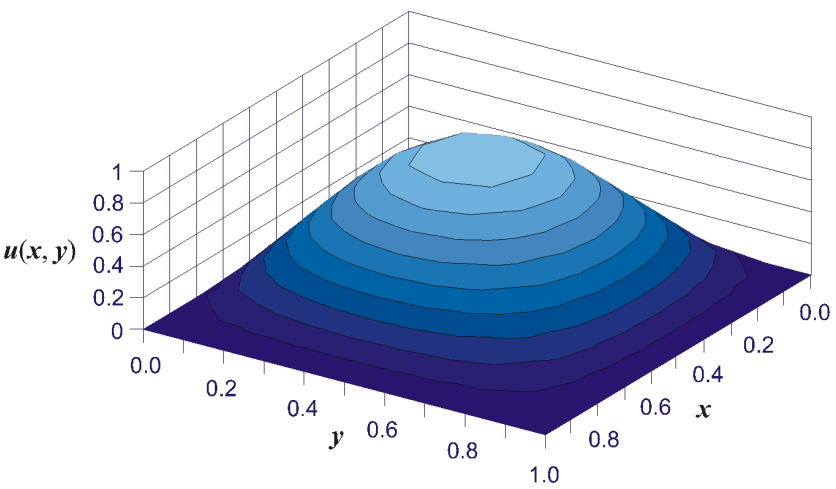

Fig. 5. The solution of the problem (15)

\section{CONCLUSIONS AND FURTHER STUDIES}

The main conclusion is that interval methods for solving partial-differential equation problems in floating-point interval arithmetic give solutions in the form of intervals which contain all possible numerical errors, i.e. representation errors, rounding errors, and errors of methods. It should be noted that for each particular problem one should choose the appropriate step sizes $h$ and $k$ to obtain the interval solution with the appropriate width.

In further studies we plan to use another (faster) exact method for solving the system of linear interval equations with the special form of band matrix occurring in the considered problem (e.g. special kind of the Cholesky method since the matrix is symmetric and positive defined or a generalization of the Crout reduction algorithm since the matrix is in a symmetric-block tridiagonal form).

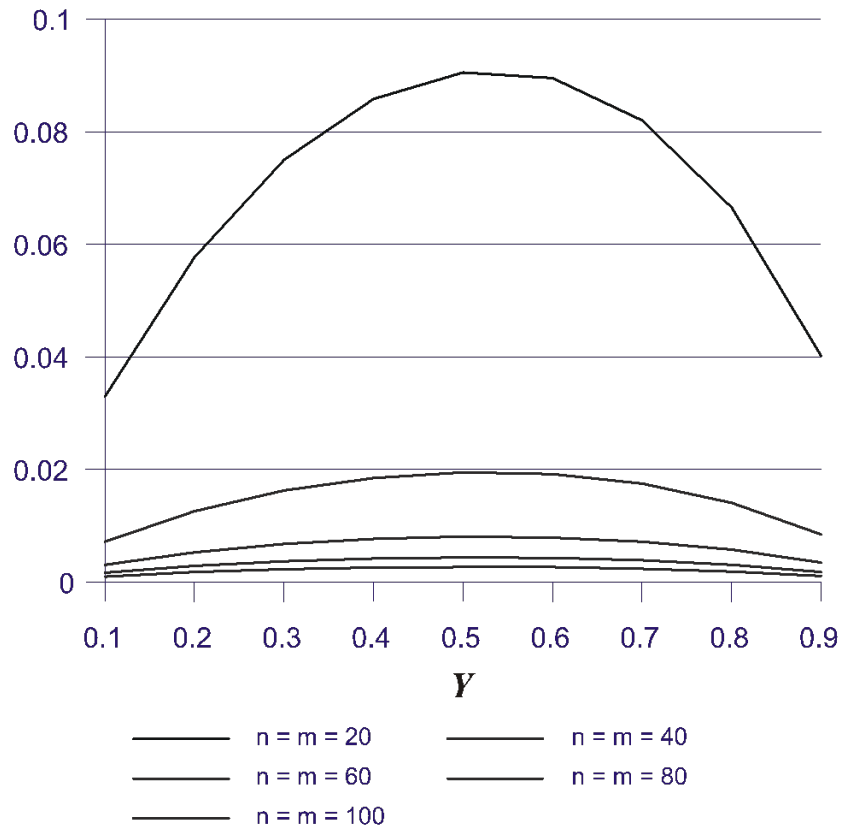

Fig. 6. The widths of interval solutions $U(X, Y)$ of the problem (15) on the grid $X=0.5$

We will try to solve other partial-differential problems (the Poisson equation is only an example of the so-called elliptic equations, and there are also partial-derivative equations 
of hyperbolic and parabolic types) using interval difference methods (not only based on the central-difference formula). It would also be interesting to construct interval methods for solving partial-differential equation problems that are based on other conventional methods, e.g. on the finite-element method.

\section{References}

[1] R.L. Burden, J. D. Faires, Numerical Analysis, $3^{\text {rd }}$ Edition, Prindle, Weber \& Schmidt, Boston 1981.

[2] R. Hammer, M. Hocks, U. Kulisch, D. Ratz, Numerical Toolbox for Verified Computing I: Basic Numerical Problems, Springer, Berlin 1993.

[3] D. Kincaid, W. Cheney, Numerical Analysis: Mathematics of Scientific Computing, $3^{\text {rd }}$ Edition, Broks/ Cole, Pacific Grove 2002.

[4] A. Marciniak, Implicit Interval Methods for Solving the Initial Value Problem, Numerical Algorithms 37, 241-251 (2004).

[5] A. Marciniak, Multistep Interval Methods of Nyström and Milne-Simpson Types, Computational Methods in Science and Technology 13(1), 23-40 (2007).

[6] A. Marciniak, On Multistep Interval Methods for Solving the Initial Value Problem, Journal of Computational and Applied Mathematics 199(2), 229-238 (2007).

[7] A. Marciniak, Selected Interval Methods for Solving the Initial Value Problem, Publishing House of Poznań University of Technology, Poznań 2009.

[8] R.E. Moore, Interval Analysis, Prentice-Hall, Englewood Cliffs 1966.

[9] J.I. Shokin, Interval Analysis [in Russian], Nauka, Novosibirsk 1982.

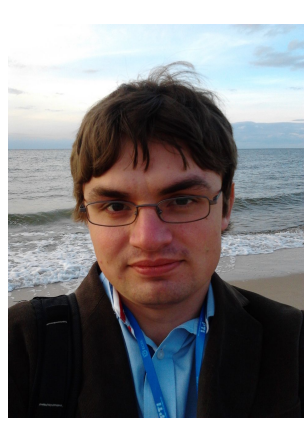

Tomasz Hoffmann was born in Piotrków Trybunalski, Poland in 1986. He is a third year PhD student in Computer Science at Poznań University of Technology. In 2010 he received his MSc degree in Computer Science and BSc degree in Mathematics at the Poznań University of Technology. His main areas of interest are: numerical methods, machine learning and information retrievial.

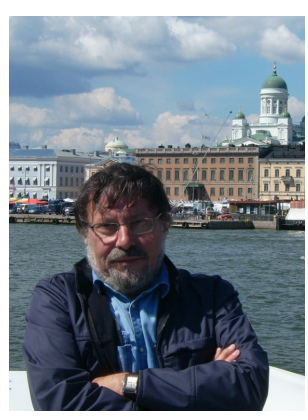

Andrzej Marciniak was born in Poznań (Poland) in 1953. He received the MSc degree in mathematics in 1977, the MSc degree in astronomy in 1979 and PhD degree in mathematics in 1981, all from the Adam Mickiewicz University in Poznań. In 1993 he received the Dr.Habil. degree in physics from the Nicolaus Copernicus University in Torun (Poland) and in 2010 he received the Professor Title from the President of Poland. From 1977 to 1987 and from 2000 to 2011 he held a research position at the Faculty of Mathematics and Computer Science of the Adam Mickiewicz University, and since 1987 he has been an assistant professor in Institute of Mathematics and then a professor of computer science at the Faculty of Computing Science of the Poznań University of Technology. From 2005 to 2008 he held the office of the President of Polish Information Processing Society. His research interests include computer programming and numerical methods, especially for solving ordinary and partial defferential equations with applications to dynamical problems. In these fields he wrote three monographs, more than 20 textbooks and a number of scientific articles. 